\title{
Multi-scale modeling of chemo-mechanical coupling in muscle contraction and applications to cardiac modeling
}

\author{
Dominique Chapelle ${ }^{a}$ \\ ${ }^{1}$ MEDISIM, Inria, Palaiseau, France
}

\begin{abstract}
We propose a muscle chemo-mechanical model by which myosin heads - that can chemically bind to actin, thus creating so-called cross-bridges producing contraction forces in sarcomeres at the subcellular level - are considered as special chemical entities having internal mechanical variables pertaining to the actual geometric configuration. This provides a thermodynamical basis for modeling the complex interplay of chemical and mechanical phenomena at the sarcomere level. The resulting model is in the form of stochastic equations governing the dynamics of these microscopic mechanical variables in a Langevin framework. Equivalently, Fokker-Planck equations can be derived to describe the evolution of the associated probability densities. Under certain assumptions the corresponding moment equations can be closed, thus directly providing access to macroscopic quantities that can be incorporated in the overall constitutive equations of the muscle tissue. The underlying thermodynamical framework also enables the derivation of compatible numerical schemes, in particular in terms of energy balances. These modeling and discretization ingredients can be integrated in a global model of the cardiac system, to represent physiological and pathological phenomena in various medical applications.
\end{abstract}

\footnotetext{
${ }^{\mathrm{a}}$ Corresponding author: dominique.chapelle@,inria.fr
} 